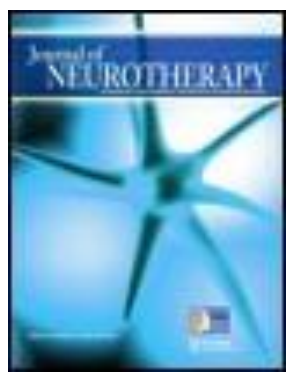

Journal of Neurotherapy: Investigations in Neuromodulation, Neurofeedback and Applied Neuroscience

\title{
Theoretical Implications of EEG Reference Choice and Related Methodology Issues
}

\author{
J. Peter Rosenfeld Associate Editor PhD ${ }^{\mathrm{a}}$ \\ a Department of Psychology , Northwestern University , 2029 Sheridan \\ Road, Evanston, IL, 60208, USA \\ Published online: 17 Oct 2008.
}

To cite this article: J. Peter Rosenfeld Associate Editor PhD (2000) Theoretical Implications of EEG Reference Choice and Related Methodology Issues, Journal of Neurotherapy:

Investigations in Neuromodulation, Neurofeedback and Applied Neuroscience, 4:2, 77-87

To link to this article: http://dx.doi.org/10.1300/J184v04n02_08

\section{PLEASE SCROLL DOWN FOR ARTICLE}

\begin{abstract}
(c) International Society for Neurofeedback and Research (ISNR), all rights reserved. This article (the "Article") may be accessed online from ISNR at no charge. The Article may be viewed online, stored in electronic or physical form, or archived for research, teaching, and private study purposes. The Article may be archived in public libraries or university libraries at the direction of said public library or university library. Any other reproduction of the Article for redistribution, sale, resale, loan, sublicensing, systematic supply, or other distribution, including both physical and electronic reproduction for such purposes, is expressly forbidden. Preparing or reproducing derivative works of this article is expressly forbidden. ISNR makes no representation or warranty as to the accuracy or completeness of any content in the Article. From 1995 to 2013 the Journal of Neurotherapy was the official publication of ISNR (Www. Isnr.org); on April 27, 2016 ISNR acquired the journal from Taylor \& Francis Group, LLC. In 2014, ISNR established its official open-access journal NeuroRegulation (ISSN: 2373-0587;

www.neuroregulation.org).
\end{abstract}

\section{THIS OPEN-ACCESS CONTENT MADE POSSIBLE BY THESE GENEROUS SPONSORS}

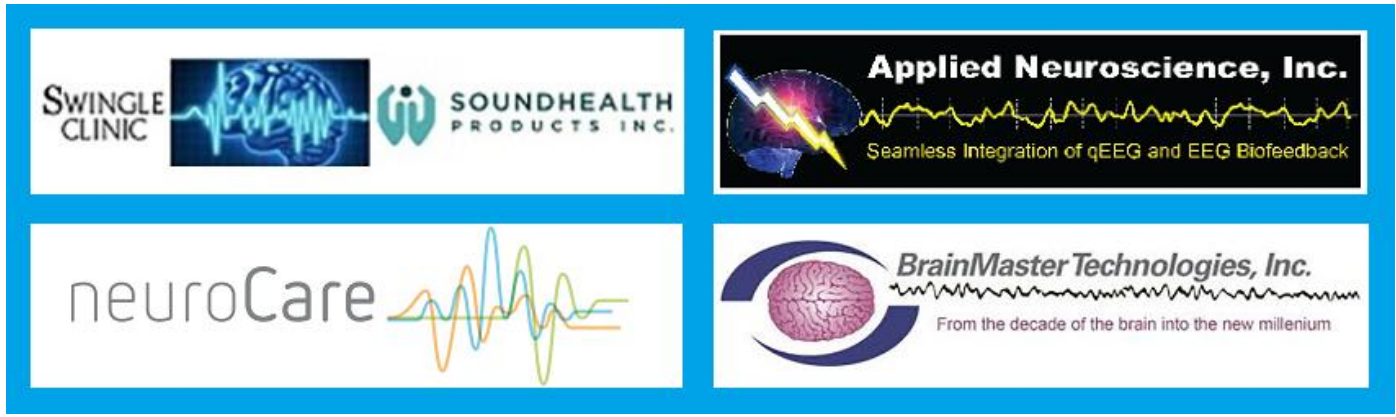




\title{
Theoretical Implications of EEG Reference Choice and Related Methodology Issues
}

\author{
J. Peter Rosenfeld, PhD, Associate Editor
}

\begin{abstract}
"Perhaps the most divisive issue among current EEG researchers is the choice of reference electrode" (Davidson, in press). There is no general agreement about appropriate references. The choice of what site to use as a reference for EEG recording usually varies with the needs of individual EEG users. As we shall see, for simple therapeutic purposes, as in EEG biofeedback (neurofeedback), the choice of reference can be straightforward: one chooses whatever montage is believed to be clinically effective. The problems associated with reference choice begin when a clinician wants to interpret apparent changes in the EEG brain map; but, perhaps not known by the clinician, the reference chosen for recording obviates such interpretation. For example, a well-established neurofeedback protocol may result in increased beta activity which seems maximal at a given site (e.g., left, parietal). However unless a proper reference was used, the observed resulting topographical
\end{abstract}

J. Peter Rosenfeld, PhD, is affiliated with the Department of Psychology, Northwestern University, 2029 Sheridan Road, Evanston, IL 60208 (E-mail: jp-rosenfeld @northwestern.edu).

The author would like to thank the following individuals in the preparation of this paper: Robert Thatcher, Emanuel Donchin, Dan Ruchkin, Richard Davidson, Joel Lubar, Jay Gunkleman, Jonathan Rosenfeld, Elsa Baehr, and Stefan Debener.

Journal of Neurotherapy, Vol. 4(2) 2000

Copyright (C) 2000 ISNR. All rights reserved. 
changes-the map-may be seriously distorted, and theoretical statements about the parts of brain involved in the therapy then become highly misleading. Although the leading researchers in the scientific EEG world have been recently converging on a position that the so-called "average electrode" reference (discussed below) is probably the best reference for all interpretive purposes, there is disagreement about how to obtain this reference montage which generally requires many electrodes $(20-128)$ that must be equally spaced all about the head (including non-scalp areas, such as the chin).

To fully appreciate these issues, some background information is required: An EEG recording from a single scalp channel requires three electrodes attached to the client (see Figure 1). One electrode is connected to the ground of the hardware system. This connection is made for safety reasons. The signals coming from the site chosen as ground should not, theoretically, have any influence on the voltage calculated as coming from the other site(s) of interest. The two other sites connected to the hardware system are differentially amplified. This means that the amplifier into which electrodes are connected calculates the difference in voltage between these sites (and amplifies that difference to make it large enough for and compatible with the computer system doing further processing).

If both sites are active, i.e., picking up brain activity, the recording is said to be bipolar (see Figure 2). In animal work, it often makes sense to record bipolar EEG because the electrodes can be accurately placed across neural tissue layers so as to maximize the localized EEG potential of interest. A classic example here is to record from the surface to the depths of a cortical gyrus-one electrode on the cortical surface, the other, one or two millimeters

FIGURE 1. The differential amplifier for this one channel subtracts the voltage at one active input from the other to yield $A C 1-A C 2$. AC1 and AC2 are connected to two scalp sites in a bipolar recording (see Figure 2). In a monopolar recording (see Figure 2), AC1 is connected to an active site of interest and AC2 is connected to theoretically "quiet" (near or at zero) reference, such as the nose in Figure 2.

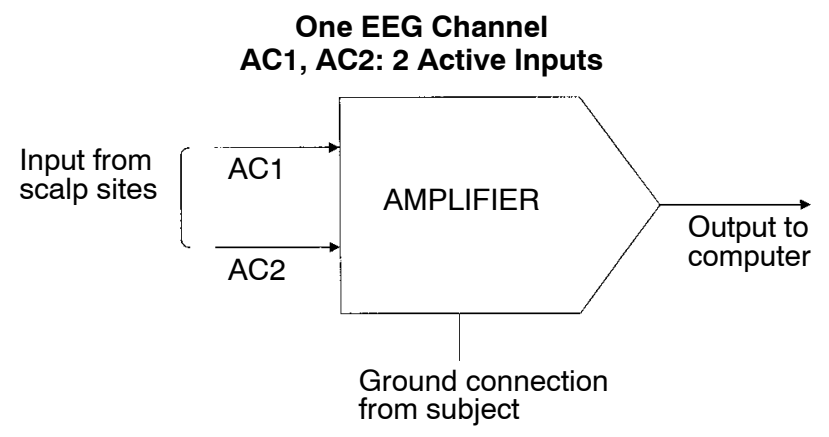


below. Obviously, this is not routinely done in humans, and most recordings are taken from the scalp. It is appreciated that some EEG biofeedback protocols are bipolar and may be effective. However, bipolar recordings from the human scalp, i.e., from 2 active scalp sites, allow no clear cut interpretations of where the action of interest is localized in brain. It may be at one active site or the other or both or in-between. This is illustrated by Table 1 which shows a hypothetical bipolar recording between $\mathrm{Cz}$ and $\mathrm{Oz}$ during a pre-treatment

FIGURE 2. Bipolar (left) vs. monopolar (right) recording. The difference between these configurations is that in the former, AC2 (see Figure 1) is connected to an active scalp site (F4), where as in the monopolar (referential) recording, AC2 is connected to the theoretically quiet nose. In both situations one earlobe (A1) is used to connect the subject to system ground.

\section{Bipolar (Left) and Monopolar (Right) Recording}
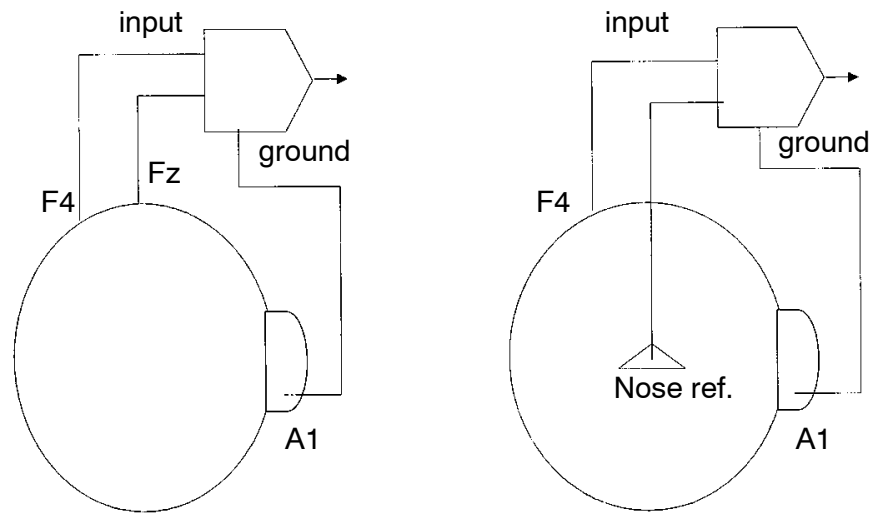

TABLE 1. Various ways bipolar recorded EEG magnitude may be altered. Units arbitrary. The "true voltages" are the voltages which would be seen if $\mathrm{Cz}$ and Oz were independently referenced to a zero voltage reference (explained in the next paragraph).

\section{True Voltage}

\begin{tabular}{lrrc} 
& $\underline{\mathrm{Cz}}$ & $\underline{\mathrm{Oz}}$ & bipolar $\mathrm{Cz}-\mathrm{Oz}$ \\
Pretreatment & 4 & 2 & 2 \\
\hline Posttreatment 1 & 6 & 2 & 4 \\
Posttreatment 2 & 4 & 0 & 4 \\
Postttreatment 3 & 8 & 4 & 4 \\
Posttreatment 4 & -4 & -8 & 4
\end{tabular}


condition and following an EEG biofeedback treatment (or whatever manipulation) designed to increase the amplitude of an EEG frequency (e.g., 10 $\mathrm{Hz}$ ). The table shows that the bipolar amplitude can be doubled in many ways: increased $\mathrm{Cz}$, no change in $\mathrm{Oz}$, decreased $\mathrm{Oz}$, no change in $\mathrm{Cz}$, various increases/decreases at both electrodes.

By far, the most typical and preferred EEG referencing montage for topographical interpretive purposes is the monpolar or referential montage ${ }^{1}$ (see Figure 2). In this configuration, instead of connecting both differential inputs to active sites (as in bipolar recording), one connects one site to a known active site of interest; i.e., a site where there are strong EEG signals (e.g., Pz), and the other site is designated reference and is connected to a head (or other body) site where there is theoretically zero EEG signal. (Some systems allow two sites, e.g., earlobes, to be connected to two separate reference inputs. However, these inputs are usually connected together internally to AC2, in Figure 1.) Thus, differences occurring over time, after treatment, etc., may be unambiguously attributed to changes at the designated active site since the reference always remains at zero.

The problem is, however, that none of the typical sites used as references by EEG workers are truly quiet, i.e., producing zero voltage. This site set includes earlobes, mastoids, nose, and physically linked ears or mastoids. These sites may contain much lower voltages than scalp sites because they are further from brain, but they are not at zero. As already noted, there is a growing consensus (Dien, 1998; Picton et al. 2000; Junghofer, Elbert, Tucker, \& Braun, 1999) based on biophysical modeling that the averaged electrode reference is the best (least biased) reference to use for EEG recording. It can be shown mathematically that if the head is modeled by a sphere within which there is a local source ${ }^{2}$ of electrical potential conducting to the entire spherical surface, a large, representative sample of surface recordings of the potentials (which are positive and negative) all around the sphere will sum (integrate) to zero, true reference neutrality. A reference of zero is, as noted, ideal.

The average electrode reference requires a minimum of twenty electrodes (Davidson, in press), and these must be equally spaced about the head (not just the scalp). The more electrodes, the better (Dien, 1998), and up to one hundred and twenty-eight electrodes have been used. Although the average reference may be the best, it too does not completely satisfy all assumptions of the model since, for one thing, the head is not a perfect sphere, nor can one sample from its undersurface through which the neck structures pass. However, if properly implemented, the average reference will provide the most accurate brain maps which most other referencing montages will distort.

It is clear that many clinical investigators do not have access to $20+$ electrode systems; indeed the "standard" EEG cap has only the nineteen sites 
for use with the 10-20 system (Jasper, 1958). Moreover, it is not likely that standard clinical equipment providers offer software capable of computing the average reference. The major EEG equipment manufacturers, who do NOT attend neurofeedback meetings, but attend the neuroscience meetings, provide software for all manner of re-montaging and QEEG, but they do not provide neurofeedback software. The alternative is to intelligently choose a more conventional reference.

A traditionally favorite reference scheme since circa 1970 is the physically linked ears/mastoids montage. This configuration may be acceptable as a reference for active midline sites $(\mathrm{Fz}, \mathrm{Cz}, \mathrm{Pz}, \mathrm{Oz})$, however for any studies in which one wants to know something about the EEG map across the entire scalp, i.e., involving frontal versus parietal, and hemispheric effects-including asymmetry-the linked ears montage is wholly inappropriate, and capable of yielding grossly distorted results. As Nunez has repeatedly argued (e.g., Nunez, 1991), “. . . it is time for the linked ears/mastoids method to die a quiet and well-deserved death." Nunez argued persuasively in just two pages why this is so, and we will not attempt to shorten his elegant presentation here. Suffice it to note that Nunez shows that if, when using a linked ears montage, the impedances for the two ears are equal-a virtually impossible condition to achieve-then linked ear voltage is $\mathrm{V}_{\mathrm{a}}$, the average of the voltage at each ear $\left(V_{1}+V_{2}\right) / 2$, where $V_{1}$ and $V_{2}$ are voltages at $A_{1}$ and $A_{2}$, respectively. This may suffice for some applications, and not distort EEG maps too much, provided the active sites of interest are not too close to the ears (e.g., $\mathrm{P}_{3}, \mathrm{Pz}$ and $\mathrm{P}_{4}$ might be acceptable but not $\mathrm{P}_{7}$ and $\mathrm{P}_{8}$ ). If however, there is significant impedance asymmetry between ear/mastoid references, which is not improbable, then any resulting brain map effects can become distorted and misleading. Nunez (1991) goes on to point out, however, that it is readily possible to compute $\mathrm{V}_{\mathrm{a}}$ : It is necessary to record the EEG with $\mathrm{A}_{1}$, one earlobe (mastoid) as reference. The other earlobe, $A_{2}$, is recorded as an active site such as $\mathrm{Cz}$. Indeed let us suppose $\mathrm{Cz}$ is the true active site used. Thus we record two channels of EEG, $\mathrm{Cz}$ referenced to $\mathrm{A} 1=\left(\mathrm{Cz}-\mathrm{A}_{1}\right)$ and $\mathrm{A}_{2}$ referenced to $A_{1}=\left(A_{2}-A_{1}\right)$. To obtain the $V_{a}$ term, for the $C z$ site, one subtracts $1 / 2\left(A_{2}-A_{1}\right)$ from $\left(C z-A_{1}\right)$. (The two terms in parentheses in the preceding sentence are the raw data recorded.) If one performs the subtraction algebraically, one obtains $\mathrm{V}_{\mathrm{a}}=\mathrm{Cz}-\left(\mathrm{A}_{2}+\mathrm{A}_{1}\right) / 2, \mathrm{Cz}$ referenced to mathematically (not physically) linked ears. One can do this for all sites, e.g., $\mathrm{F}_{2}, \mathrm{~F}_{3}, \mathrm{~F}_{4}, \mathrm{C}_{3}, \mathrm{C}_{4}, \mathrm{P}_{3}, \mathrm{P}_{\mathrm{z}}, \mathrm{P}_{4}$, etc. The mathematical off-line linkage avoids most of the pitfalls associated with physical linkage.

I anticipate the following question having arisen in readers' minds during reading the previous paragraph: "If we already have $\left(\mathrm{Cz}-\mathrm{A}_{1}\right), \mathrm{Cz}$ referenced to one ear, isn't that good enough? Isn't a single ear reference, also a typical reference, OK?" If $\mathrm{Cz}$ or other midline sites are the sites of interest, 
the answer may well be yes. But if the sites of interest are laterally distributed, e.g., $\mathrm{C}_{5}, \mathrm{C}_{6}, \mathrm{C}_{7}, \mathrm{C}_{8}$, etc., the answer is no. That is because scalp site voltage near the unilateral reference site, $\mathrm{A}_{1}$, will be attenuated more than sites far contralaterally from $\mathrm{A}_{1}$. The resulting voltage map or EEG asymmetry estimates are distorted. Here is where the interests of the EEG worker and common sense come into play: If one is interested only in asymmetry between pairs of homologeous electrodes (e.g., F3 and F4), then one can use either the averaged ears reference, $V_{a}$, if one has means for its computation, ${ }^{3}$ or one could use the nose as a reference site (as is not at all uncommon, e.g., Graae, Tenke, Bruder et al., 1996). It is equidistant from the homologous site pairs and should be no source of bias within a pair. Obviously, however, if one were interested in frontal vs. parietal effects, the nose reference would attenuate the former voltages more than the latter due to its greater proximity to frontal leads. In this situation, a single earlobe would be better. I would suggest using the left ear for right frontal versus right parietal (or occipital) comparisons, and the right ear for left frontal-parietal comparisons. Mathematically linked ears $\left(\mathrm{V}_{\mathrm{a}}\right)$ is likely the best reference in this situation, notwithstanding that the average reference, discussed above, is probably the least biased of all in most situations.

All the preceding discussion suggests using one site as reference "online," i.e., during recording, because one can then mathematically "re-montage" the data off-line so that all possible referencing schemes are derivable and comparable. It doesn't matter which single site is chosen for on-line referencing. Workers should urge their vendors to provide re-montaging software patches. The compelling aspect of this plan is that if a certain neurofeedback protocol or database specifies, say, $\mathrm{A}_{1}$ as the single site reference, a clinician can go ahead and use it for neurofeedback or database comparison, but then, as long as one or two $\left(\mathrm{A}_{2}\right.$, nose) other relatively quiet sites are recorded as active sites, the relatively undistorted actual map effects can be obtained by re-montaging off-line (after the recording/treatment session). If the electrode density $>20$, even the least biased, average electrode reference may be computed (see Dien, 1998), as well all other montages. Effects on the actual maps can then be compared across montages, and if most derived unbiased montages yield similar maps, one can state with some confidence where the localization of effects are. The critical point here, is that one can not re-montage if one has used the physically-linked ear montage as reference during recording.

This brings us to the situation where one has used a physically-linked ears (or other inappropriate) reference because the clinically effective protocol called for it, or because one wishes to compare data to a normative database obtained with linked ears. Very simply, there is nothing amiss about such treatment procedures, but one should not make any statements regarding 
localization of effects. If a database (based on a poor reference) allows valid discrimination among client types, then one may safely use it, but only for diagnostic purposes, not as a model of where in brain pathology is localized. Indeed, the database author himself should refrain from interpreting inappropriately referenced map effects. Similarly, the protocol developer who has used a reference inappropriate for interpreting localization effects should refrain from commenting on mechanism. This caveat applies to no one as strongly as it applies to the present author.

Rosenfeld and colleagues (Rosenfeld, 2000) have developed a neurofeedback protocol for treatment of affective disorders which uses sites $F_{4}$ and $F_{3}$, both "referenced" to Cz. We use this configuration because it is the one which Davidson (1995) used in his early demonstrations of the relationship of frontal activation asymmetry and emotion. We train people to increase the $\left(\mathrm{F}_{4}-\mathrm{Cz}\right)$ alpha output and decrease the $\left(\mathrm{F}_{3}-\mathrm{Cz}\right)$ output. (The rationale is in Rosenfeld, 2000.) We have found the protocol to be clinically effective, so it is reasonable to keep using it, but we really can have no idea how, where, or why it works from our bipolar, Cz-referenced data. There are at least two reasons why this is so.

(1) $\mathrm{Cz}$ is a particularly terrible reference for topographical interpretation because it is electrically active. The formula typically used to calculate the frontal alpha asymmetry score is one of these two:

$$
\begin{aligned}
& \text { (1) } \mathrm{A}_{\mathrm{A}}=\left(\mathrm{F}_{4}-\mathrm{F}_{3}\right) /\left(\mathrm{F}_{4}+\mathrm{F}_{3}\right) \\
& \text { (2) } \mathrm{A}_{\mathrm{B}}=\log \left(\mathrm{F}_{4}\right)-\log \left(\mathrm{F}_{3}\right)
\end{aligned}
$$

where $F_{4}$ and $F_{3}$ are alpha power or magnitude at right and left frontal scalp sites according to the 10-20 system (Jasper, 1958). (Although $A_{A}$ and $A_{B}$ are not mathematically equivalent, they are highly correlated, as we have reported empirically in Rosenfeld, 2000, and as we will model below.) Looking at these formulae suggests that the reference electrode is irrelevant to the calculations of $A_{A}$ and $A_{B}$, but it must be recalled that any EEG channel recording is the difference between the active site of interest $\left(F_{4}\right.$ or $\left.F_{3}\right)$ and the reference electrode, which we will notate as R. Thus the above formulae are in fact:

$$
\begin{aligned}
& \text { (1A) } A_{A}=\left[\left(F_{4}-R\right)-\left(F_{3}-R\right)\right] /\left[\left(F_{4}-R\right)+\left(F_{3}-R\right)\right] \\
& \text { (1B) } A_{B}=\log \left(F_{4}-R\right)-\log \left(F_{3}-R\right)
\end{aligned}
$$

Now if $R$ is a truly quiet reference (= 0 signal), then formulae (1A) and (1B) reduce to (1) and (2). But if $\mathrm{R}$ is an active source of voltage as is the case when $\mathrm{Cz}$ is $\mathrm{R}$, then formula $1 \mathrm{~A}$ becomes: 


$$
A_{A}=\left[\left(F_{4}-C z\right)-\left(F_{3}-C z\right)\right] /\left[\left(F_{4}-C z\right)+\left(F_{3}-C z\right)\right]=\left[F_{4}-F_{3}\right] /\left[F_{4}+F_{3}-2 C z\right]
$$

Inspection of this formula makes it clear that from one time to another, $\mathrm{F}_{3}$ and $\mathrm{F}_{4}$ can stay constant, while changes at $\mathrm{Cz}$ alone can change the asymmetry score. This is illustrated in Table 2 , where $F_{4}$ and $F_{3}$ were held constant at 40 and $50 \mu \mathrm{V}$, respectively, and $\mathrm{Cz}$ was systematically varied from -50 to + $35 \mu \mathrm{V}$ in $5 \mu \mathrm{V}$ steps. Also shown in Table 2 is $\log \left(\mathrm{F}_{4}-\mathrm{Cz}\right)-\log \left(\mathrm{F}_{3}-\right.$ $\mathrm{Cz}$ ) as $\mathrm{Cz}$ varies; the $\mathrm{A}_{\mathrm{B}}$ score. It is evident that asymmetry scores (both $\mathrm{A}_{\mathrm{A}}$ and $A_{B}$ ) can change entirely due to changes at the $\mathrm{Cz}$ reference, and that if a quiet reference (approaching zero) were used, there would be no changes in real asymmetry, as are the actual cases modeled in Table 2. (By the way, the correlation of $\mathrm{A}_{\mathrm{A}}$ and $\mathrm{A}_{\mathrm{B}}$ in Table 2 is 9992 for the 19 cases shown.)

\section{TABLE 2}

\begin{tabular}{lllll} 
& True Voltages, $\mu \mathrm{V}$ & \multicolumn{2}{c}{ Asymmetry Scores } \\
$\mathrm{F}_{4}$ & $\mathrm{~F}_{3}$ & $\mathrm{Cz}$ & \multicolumn{1}{c}{$\mathrm{A}_{\mathrm{A}}$} & \multicolumn{1}{c}{$\mathrm{A}_{\mathrm{B}}$} \\
\hline 40 & 50 & -50 & -0.05263 & -0.04576 \\
40 & 50 & -45 & -0.05556 & -0.0483 \\
40 & 50 & -40 & -0.05882 & -0.05115 \\
40 & 50 & -35 & -0.0625 & -0.05436 \\
40 & 50 & -30 & -0.06667 & -0.05799 \\
40 & 50 & -25 & -0.07143 & -0.06215 \\
40 & 50 & -20 & -0.07692 & -0.06695 \\
40 & 50 & -15 & -0.08333 & -0.07255 \\
40 & 50 & -10 & -0.09091 & -0.07918 \\
40 & 50 & -5 & -0.1 & -0.08715 \\
40 & 50 & 0 & -0.11111 & -0.09691 \\
40 & 50 & 5 & -0.125 & -0.10914 \\
40 & 50 & 10 & -0.14286 & -0.12494 \\
40 & 50 & 15 & -0.16667 & -0.14613 \\
40 & 50 & 20 & -0.2 & -0.17609 \\
40 & 50 & 25 & -0.25 & -0.22185 \\
40 & 50 & 30 & -0.33333 & -0.30103 \\
40 & 50 & 35 & -0.5 & -0.47712
\end{tabular}

NOTE: As F4 and F3 remain constant, but as $\mathrm{Cz}$ systematically varies, both asymmetry scores $A_{A}$ and $A_{B}$ change, and their correlation is .9992 
Table 3 is similar to Table 2, except that $\mathrm{Cz}$ is held constant as $\mathrm{F}_{4}$ and/or $\mathrm{F}_{3}$ are varied. The comparable changes in $\mathrm{A}_{\mathrm{A}}$ shown could be attributed to changes either at $\mathrm{F}_{3}$ and/or $\mathrm{F}_{4}$. Another case, not shown, would involve changes of various sizes in all three variables, $\mathrm{F}_{4}, \mathrm{~F}_{3}$, and $\mathrm{Cz}$. The reader skilled in spread sheet algebra can amuse him/herself exploring the various changes in asymmetry of comparable amounts, which cannot be logically attributed to changes in activity at any subset or set of these sites.

(2) It is also possible that alpha at $\mathrm{F}_{4}$ and $\mathrm{F}_{3}$ could be of exactly the same amplitude, but out of phase, and with the phase relationship continually changing such that one frontal site will lead the other most of the time. This phase asymmetry (and consequently compromised coherence) could appear as (false) amplitude asymmetry.

Thus our asymmetry paradigm is deeply flawed in terms of our ability to understand where and what kind of changes are occurring in which cortical areas, yet it can be effectively used clinically. The only reason we keep suggesting that amplitude asymmetry mechanisms at $\mathrm{F}_{3}$ and $\mathrm{F}_{4}$ underlie our effects is because Davidson and others continue to find the asymmetry-emotion correlations with various reference montages, including the average electrode montage.

There is yet another reason, not relating directly to reference choice, why inferences about localization of cortical changes can not be legitimately made on the basis of scalp recordings: The human brain has gyri ("hills") and sulci (valleys) in the cortical layers. Figure 3, showing one neuron with an electrical field oriented from the top down has its field perpendicular to the scalp and cortical surfaces. It is shown as if it is in the midpoint of a gyrus. However, as the cortical surface folds down into a sulcus, the same kind of field now becomes parallel to the scalp surface, and it is intuitively obvious that the field will be volume-conducted to the cortical surface in a different manner than as in Figure 2. The simplified bottom line is that just because a particular EEG effect is largest at, say, Fz, does not allow the conclusion that

\section{TABLE 3}

\begin{tabular}{lllll} 
Time & $\mathrm{F}_{4}$ & $\mathrm{~F}_{3}$ & $\mathrm{Cz}$ & $\mathrm{A}_{\mathrm{A}}$ \\
\hline 1 & 5 & 4 & 1 & .125 \\
$2 \mathrm{a}$ & $6^{\star}$ & 4 & 1 & .200 \\
$2 \mathrm{~b}$ & 5 & $3^{*}$ & 1 & .250 \\
$2 \mathrm{c}$ & $5.5^{\star}$ & $3^{*}$ & 1 & .278
\end{tabular}

NOTE: Cz stays constant at 1 from Time 1 to three other times, $2 a, 2 b, 2 c$ involving changes (shown with asterisks*) in $F_{4}, F_{3}$, and both $F_{4}$ and $F_{3}$. All three second times show comparable changes (increases) in $\mathrm{A}_{\mathrm{A}}$ scores. 
FIGURE 3. Synaptic depolarizing transmitter at apical dendrites causes positive ions to enter the dendrites leaving a negatively charged wake near the cortical surface. The deeper cell body then extrudes positive charge producing a positive area. Positive charge current thus flows from the depths to the surface of cortex. The difference in potential exists between the source and sink for the current. This voltage is differentially conducted to differing points on the head. (There are other ways that other voltages/currents can be generated in cortex, e.g., hyperpolarization vs. depolarization at the apical dendrite.)

\section{Origin of Local Cortical Potential Field}

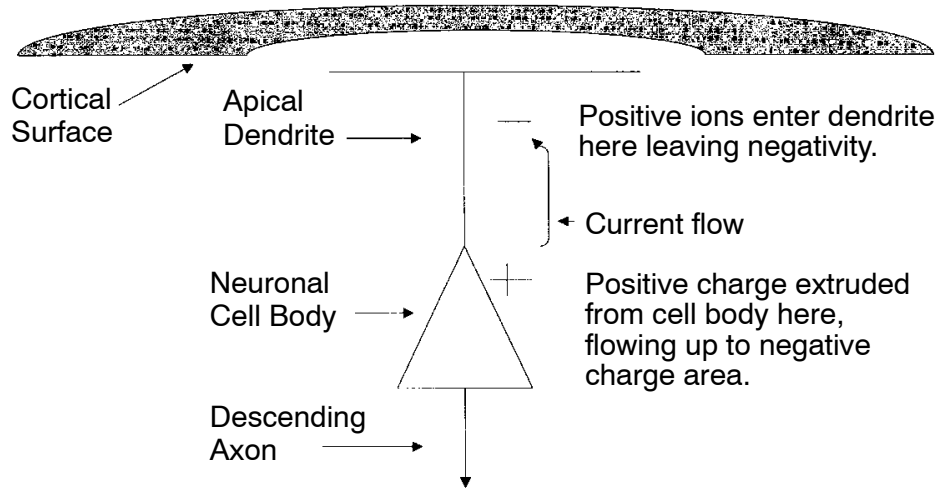

the cortical source of this activity is directly below Fz. As Cook et al. (1998) put it, "A number of investigators have cautioned that since EEG activity recorded at a single electrode may arise from local or distant sources, topographic maps may not accurately characterize local brain function." In depth discussion of such phenomena may be found in Nunez (1995), Cook et al. (1998).

I thus urge clinical investigators to carefully temper statements made in publications about localization of effects, unless appropriate referencing was used.

\section{NOTES}

1. It is emphasized that we are speaking of topographic interpretation. For certain clinical purposes (e.g., seizure detection), bipolar montages are also recommended by the American Association of Electroneurodiagnostic Technologists (ASET). Their guidelines may be found on their web site: www.aset.org/. Also, Cook et al. (1998) have shown that highly specialized, closely spaced bipolar recordings can help localize cerebral perfusion, one measure of activity. 
2. It is noted that a "local source of electrical potential" is generated in a cortical area during synchronous synaptic transmission, as, for example, when the apical dendrites closest to the surface of cortex are depolarized and positive current in the form of sodium ions, enter the dendrite leaving a negative area in their wake (see Figure 3). The lower portion of the neuron, the cell body, will extrude positivity as the positive current flows from depth to surface dendrites, re-supplying the apical influx of positivity. The upshot is that an electrical field-a difference in potential-will exist from surface to depths of cortex in all such functionally related neurons in the local area of the field. It is this difference in potential which defines a "local source of electrical potential," which will appear negative at the scalp overlying the surface negative side of the field, and both positive and negative elsewhere on the surface of the head.

3. As shown above, it is a simple computation, but many equipment providers do not include this feature.

\section{REFERENCES}

Cook, I.A., O’Hara, R., Sebastian, H.J. et al. (1998). Assessing the accuracy of topographic EEG mapping for determining local brain function. Electroencephalography and Clinical Neurophysiology, 107, 408-414.

Davidson, R.J. (1995). Cerebral asymmetry, emotion, and affective style. In: Davidson, R.J., Hugdahl, K. (eds). Brain Asymmetry. (pp. 361-387). Cambridge: M.I.T. Press.

Davidson, R.J., Jackson, D. \& Larson, C. (In press). Human electroencephalography. In J.T. Cacioppo, G. Bernston, \& L. Tassinary (Eds.), Principles of Psychophysiology (2nd Edition). New York: Cambridge University Press.

Dien, J. (1998). Issues in the application of the average reference: Review, critiques, and recommendations. Behavioral Research Methods, Instruments, and Computers, 30(1), 34-43.

Graae, F., Tenke, C., Bruder, G. et al. (1996). Abnormality of EEG alpha asymmetry in female adolescent suicide attempters. Biol Psychiatry, 40, 706-713.

Jasper, H.H. (1958). Report of the committee on methods of clinical examination in electroencephalography. Electroencephalography and Clinical Neurophysiology, 10, 370-375.

Junghofer, M., Elbert, T., Tucker, D.T., \& Braun, C. (1999). The polar average reference effect. Clinical Neurophysiology, 110, 1149-1155.

Nunez, P. (1991). Comments on the paper by Miller, Lutzenberger, and Elbert. Journal of Psychophysiology, 5, 279-280.

Nunez, P. (1995). Neocortical Dynamics and Human EEG Rhythms. (pp. 14-18, 48-54, ff.) NY: Oxford University Press.

Picton, T.W., Bentin, S., Berg, P., Donchin, E., Hillyard, S.A., Johnson, Jr., R., Miller, G.A., Ritter, W., Ruchkin, D.S., Rugg, M.D., \& Taylor, M.J. (In press). Guidelines for using human event-related potentials to study cognition. Recording standards and publication criteria. Psychophysiology, 37, 127-152.

Rosenfeld, J.P. (2000). An EEG biofeedback protocol for affective disorders. Clinical Electroencephalography, 31, 7-12. 